\title{
Segmentation of the Thalamus in Multi-Spectral MR Images Using a Combination of Atlas-Based and Gradient Graph Cut Methods
}

\author{
Ryan Datteri $^{a}$, Christian Barillot ${ }^{b}$, Benoit M. Dawant ${ }^{a}$, and Jeremy Lecoeur ${ }^{b}$ \\ ${ }^{a}$ Department of Electrical Engineering and Computer Science, Vanderbilt University; Nashville, TN \\ 37235, USA; \\ ${ }^{b}$ VisAGeS team, INRIA, IRISA, CNRS, INSERM, Rennes, France
}

\begin{abstract}
Two popular segmentation methods used today are atlas based and graph cut based segmentation techniques. The atlas based method deforms a manually segmented image onto a target image, resulting in an automatic segmentation. The graph cut segmentation method utilizes the graph cut paradigm by treating image segmentation as a max-flow problem. A specialized form of this algorithm was developed by Lecoeur et al [1], called the spectral graph cut algorithm. The goal of this paper is to combine both of these methods, creating a more stable atlas based segmentation algorithm that is less sensitive to the initial manual segmentation. The registration algorithm is used to automate and initialize the spectral graph cut algorithm as well as add needed spatial information, while the spectral graph cut algorithm is used to increase the robustness of the atlas method. To calculate the sensitivity of the algorithms, the initial manual segmentation of the atlas was both dilated and eroded $2 \mathrm{~mm}$ and the segmentation results were calculated. Results show that the atlas based segmentation segments the thalamus well with an average Dice Similarity Coefficient (DSC) of 0.87 . The spectral graph cut method shows similar results with an average DSC measure of 0.88 , with no statistical difference between the two methods. The atlas based method's DSC value, however, was reduced to 0.76 and 0.67 when dilated and eroded respectively, while the combined method retained a DSC value of 0.81 and 0.74 , with a statistical difference found between the two methods.
\end{abstract}

Keywords: ABA, non-rigid registration, thalamus segmentation, graph cut, spectral gradient

\section{INTRODUCTION}

Segmentation is a useful tool in medical imaging and is used, for example, in computer-guided surgery [2]. The thalamus can be a difficult organ to segment since there is little intensity gradient between it and surrounding structures. Therefore, we have used an atlas based segmentation method since it utilizes the spatial relationship found within images and a priori information from the atlas image. However, registration errors, differences in anatomy, and mistakes in the initial manual segmentation of the atlas, though not common, may cause errors in the final segmentation. To address this issue, we have complemented the atlas based method with a spectral gradient graph cut and we have evaluated the robustness of this combined approach. The spectral gradient method is a technique that merges different image modalities into the standard graph cut paradigm.

An automatic segmentation of the thalamus may be useful for the computer-aided planning of Deep Brain Stimulation (DBS) procedures and other groups have segmented the thalamus both semi-automatically [3] and automatically [4][5][6][7]. Heckenberg et al. [3] utilized a manually selected seed point to initialize a Lagrangian Surface Flow algorithm. The Lagrangian Surface flow algorithm is trained from a dataset and a model is grown from the seed-point to segment the thalamus. Initial results looked promising but only included one image segmented. Pohl et al. [4] utilized a registration method, segmentation, and an image artifact estimation method along with expectation maximization to segment 22 cases. The group trained a model that was utilized to restrict the registration and resulting segmentation. Akhoundi-Asl [5] also created a model of the thalami but utilized the left and right thalamus both individually and by coupling both organs for the model creation. Then, the authors utilized an entropy based nonparametric method to segment the thalami within the parameters created from the model. The authors found that coupling the two thalami resulted in a better segmentation than by creating individual models for the left and right thalamus. In [6] Akhoundi-Asl 
utilized a probabilistic anatomical atlas and image information to aggregate similar voxels into tissue classes and segmentations. Moghaddam et al. [7] developed a thalamus segmentation method that described the structure of the
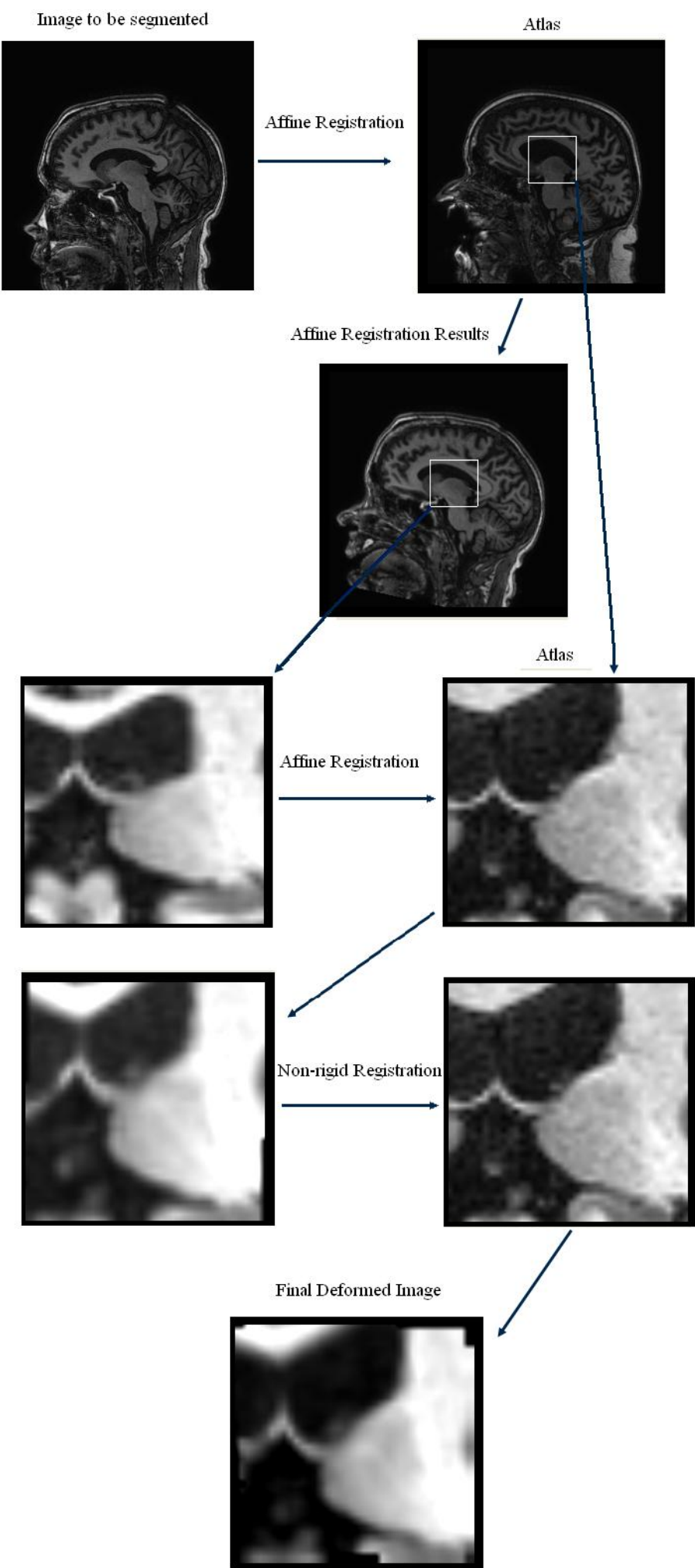

Figure 1: The registration process of the atlas based segmentation method thalamus using geometric moment invariants. Then, utilizing these features, the group trained an Artificial Neural Network which was used to classify each voxel as either inside the thalamus or outside. Here, we propose an alternative approach. To the best of our knowledge, no group has utilized a registration method combined with a spectral graph cut method to segment the thalami from MR images.

\section{METHODS}

\subsection{Images}

This study contains 11 sets of image volumes obtained with IRB approval. One of the volumes was chosen as an atlas leaving ten images to segment. Both the left and right thalamus were segmented, totaling 20 individual segmentations. All of the images used in the study were acquired on a 3 Tesla Philips scanner. The T1-weighted images have 256x256x170 1mm isometric voxels, and were acquired with the parameters $\mathrm{TE}=3.65$, $\mathrm{TR}=7.92$ and an imaging frequency of 127.8. The T2-weighted images are $512 \times 512 \times 45$ with voxel sizes $0.46875 \times 0.46875 \times 2$ and were acquired with the parameters $\mathrm{TE}=80$ and $\mathrm{TR}=3000$. The ground truth manual segmentations were drawn on the T1-weighted images.

\subsection{Atlas Based Registration}

We first manually delineated the thalamus in an atlas image. Then, a global affine registration is computed and used to project the target image that we want to segment onto the atlas image. A predefined bounding box is extracted from both the atlas and target image. Another affine registration is performed locally between these two boxes, again resulting in a transformation that is used to project the target onto the atlas image. This second affine registration is performed to limit the registration on a local area within the image. The size of the box is determined by the size and shape of the thalamus within the atlas image, with an arbitrary amount of padding to aid in the local affine registration. For the thalamus a region of $60 \times 40 \times 40$ voxels was chosen for the local registration box.

A local non-rigid registration [8] is then performed between the results of the local affine registration and the atlas image (see Figure 1). The manual contours drawn on the atlas are then projected onto 
the target image utilizing the deformation fields that were the result of the three registrations.

The non-rigid registration approach is an algorithm called ABA for adaptive bases algorithm [8]. This algorithm uses normalized mutual information [9] described in (1) as the similarity measure and models the deformation field that registers the two images as a linear combination of radial basis functions (2) with finite support.

$$
\begin{gathered}
\mathrm{NMI}=\frac{\mathrm{H}(\mathrm{A})+\mathrm{H}(\mathrm{B})}{\mathrm{H}(\mathrm{A}, \mathrm{B})} \\
v(x)=\sum_{i=1}^{N} C i \Phi(X-X i)
\end{gathered}
$$

Where $\mathrm{x}$ is a coordinate vector, $\Phi$ is one of Wu's compactly supported positive radial basis functions [10], and the $\mathbf{c}_{i}{ }^{\prime} \mathrm{s}$ are the coefficients of these basis functions. The goal is to find the $\mathbf{c}_{i}$ 's that maximize the mutual information between the images. The optimization process for the coefficients includes a steepest gradient descent algorithm combined with a line minimization algorithm. The steepest gradient descent algorithm determines the direction of the optimization. The line minimization calculates the optimal step in this direction.

One feature that distinguishes this algorithm from others (for example, Rueckert et al [11]) is the fact that we do not work on a regular grid. Rather, areas of difference are identified and the deformation field is adjusted only on these identified regions. This is done as follows. A regular grid of basis function is placed on the images. The gradient of the similarity measure with respect to the coefficients of the basis functions is then computed. The location of the basis functions for which this gradient is above a predetermined threshold is used to determine areas of mismatch. The rationale for this choice is that if the gradient is low, either the images are matched well because we have reached a maximum or the information content in this region is low. In either case, trying to modify the transormation in these regions is not productive. Optimization is then performed locally on the identified regions (for more details, refer to Rohde et al [11]).

Both the forward and the backward transformations are computed simultaneously, and the transformations are constrained to be inverses of each other using the method proposed by Burr [12]. Although this cannot be proven analytically, experience has shown that the inverse consistency error achieved with this approach is below the voxels' dimension. In our experience, enforcing inverse consistency improves the smoothness and regularity of the transformations.

One important objective of a non-rigid registration algorithm is to produce transformations that are topologically correct (i.e., transformations that do not include tearing or folding). This is difficult to guarantee and it is often implemented by constraining the transformation (e.g., adding a penalty term that is proportional to the second derivative of the deformations field [11]). Here, we follow the same approach, but the field is regularized by constraining the difference between the coefficients of adjacent basis functions (the $\mathbf{c}_{i}{ }^{\prime} \mathrm{s}$ ) using a threshold $\varepsilon$. The concept is simple: if the coefficients of adjacent basis functions vary widely, the resulting deformation field changes rapidly. This can be useful as it permits computing transformations that require large local displacements but it may also produce transformations that are ill-behaved. Thus, the threshold $\varepsilon$ can be used to control the regularity and the stiffness properties of the transformation. Small values produce smooth transformations that are relatively stiff. Large values lead to transformations that are more elastic but less regular. The threshold value for the non-rigid registration used in the atlas based segmentation method utilized a threshold value of $\varepsilon=0.2$, a relatively large threshold.

\subsection{Spectral Gradient Based Graph Cuts}

According to the scheme developed by Boykov et al. [13][14], the segmentation problem is described by a directional flow graph which represents the image. The node set is defined by two particular nodes called terminal nodes - also known as "source" and "sink" - which respectively represent the classes "object" and "background", the other nodes being the 3D volume voxels. The directed weighted edges connect each of the nodes, encoding the similarity between the two considered voxels. A segmentation then, is the minimum cut that can be made on the directional flow graph. 
Let the set $P$ contain all the voxels $p$ of an image, let the set $N$ be all pairs $\{\mathrm{p}, \mathrm{q}\}$ where $\{\mathrm{p}, \mathrm{q}\}$ are neighboring elements of $P$, and let $\mathrm{V}=\left(\mathrm{V}_{1}, \mathrm{~V}_{2}, \ldots, \mathrm{V}_{\mathrm{p}}\right)$ be a binary vector where each $\mathrm{V}_{\mathrm{p}}$ can be labeled either "object" or "background", thus defining a segmentation. The energy we want to minimize by the graph cut is given by (3):

$$
E(V)=\alpha \cdot \sum_{p \in P}\left(V_{p}\right)+\sum_{\substack{\{p, q\} \in N \\ V_{p} \neq V_{q}}} B_{\{p, q\}}
$$

The term $R_{p .}($ ), commonly referred to as the regional term, expresses how the voxel $p$ fits into given models of the object and background. The term $\mathrm{B}\{\mathrm{p}, \mathrm{q}\}$, known as the boundary term, reflects the similarity of the voxels $p$ and $q$. Hence, it is large when $p$ and $q$ are similar and close to zero when they are very different. For the graph cut algorithm, the regional term is represented by what are called t-links while the boundary term is represented by what are called n-links. The coefficient $\alpha$ is used to adjust the importance of the region and boundary terms.

Based on the work from Lecoeur et al. [1], a color image is created by combining the T1 and T2 images along with the results of the atlas based segmentation. Each image is treated as one of the color channels in a RGB color image (i.e. T1 $=$ red, $\mathrm{T} 2=$ green, atlas segmentation $=$ blue). The atlas segmentation image is a gray-level image; the details on how it is formed will be discussed later.

Utilizing the newly created color image, we calculate the spectral gradient. The spectral gradient, introduced by Geusebroek et al. [15], is based on the psycho-visual color theory and on Koenderink's Gaussian derivative color model [16]. The spectral intensity (e) of an image is based on the idea that color can be interpreted by the intensity in which it falls onto the retina. Geusebroek et al. have shown that the spectral gradient can be approximated by multiplying the RGB values of the image by two specific matrices (4).

$$
\left(\begin{array}{c}
e \\
e_{\lambda} \\
e_{\lambda \lambda}
\end{array}\right)=\left(\begin{array}{ccc}
-0.019 & 0.048 & 0.011 \\
0.019 & 0 & -0.016 \\
0.047 & -0.052 & 0
\end{array}\right) \cdot\left(\begin{array}{ccc}
0.621 & 0.133 & 0.194 \\
0.297 & 0.563 & 0.049 \\
-0.009 & 0.027 & 1.105
\end{array}\right) \cdot\left(\begin{array}{l}
R \\
G \\
B
\end{array}\right)
$$

The application of the spectral gradient approximation on our combined color image gives us the spectral intensity as well as the first and second order derivative of the spectral intensity $\left(e, e_{\lambda}\right.$, and $\left.e_{\lambda \lambda}\right)$. Once the spectral intensity and its derivatives are computed, we can use the following differential properties to calculate the contrast of the spectral intensity and its derivatives.

$$
\begin{aligned}
\varepsilon & =\frac{1}{e} \cdot \frac{\partial e}{\partial \lambda}=\frac{e_{\lambda}}{e} \\
\varepsilon_{\lambda} & =\frac{\partial \varepsilon}{\partial \lambda}=\frac{e \cdot e_{\lambda \lambda}-e_{\lambda}^{2}}{e^{2}}
\end{aligned}
$$

Then, (5) and (6) are utilized to compute the n-links for the graph cut (7).

$$
B_{\{p, q\}}=\alpha \cdot \exp \left(-\frac{1}{2} \cdot \frac{[\varepsilon(p)-\varepsilon(q)]^{2}+\left[\varepsilon_{\lambda}(p)-\varepsilon_{\lambda}\right]^{2}}{\sigma^{2}}\right) \cdot \frac{1}{\operatorname{dist}(p, q)}
$$


Where $p$ and $q$ are two neighboring voxels and $\sigma$ is a normalization factor. The t-links on the other hand are created based on the negative log-likelihood that the target voxel belongs to the group of "object" or "background". The base group of "object" and "background' voxels are chosen manually or, in our case, by the atlas segmentation. Therefore the t-link to the source is $\infty$ if it has already been chosen as an "object" voxel and the t-link to the sink is $\infty$ if the voxel has been chosen as a "background" voxel. If the voxel has not been predefined, the value of the t-link is the negative loglikelihood that the voxel belongs to the "object" and the "background" group. The value for the t-link to the source is defined by (8) while the value for the t-link to the sink is defined in (9).

$$
\begin{aligned}
& R_{p}(O)=-\ln P\left(\Psi_{p} \mid B\right) \\
& R_{p}(B)=-\ln \mathbb{P}\left(\Psi_{\mathrm{p}} \mid O\right)
\end{aligned}
$$

Where $\Psi_{\mathrm{p}}$ is the vector containing the intensity of voxel $p$ in the three images used to build the spectral image. From each set of pre-defined seeds (object and background), we calculate the mean vector $\overline{\Psi_{c}}$ and the covariance matrix $\sum_{\mathrm{c}} . P$ is defined as the class-membership probability of a voxel $v$, computed with the multivariate normal distribution formula as shown in (10).

$$
P\left(\Psi_{\mathrm{v}} \mid c\right)=\exp \left[-\frac{1}{2}\left(\Psi_{\mathrm{v}}-\overline{\Psi_{\mathrm{c}}}\right)^{\mathrm{T}} \cdot \Sigma_{\mathrm{c}}^{-1} \cdot\left(\Psi_{\mathrm{v}}-\overline{\Psi_{\mathrm{c}}}\right)\right]
$$

After the graph is created, a graph cut [14] is performed resulting in a segmentation.

In an effort to reduce the amount of time taken to perform the graph cut we utilized a hierarchical scheme. This method, first developed by Lombaert et al., [17] is inspired by the multilevel graph partition technique [18] and the narrow band from level sets [19]. The method first computes the graph cut on the coarsest level and in again in the successive higher level, but only on a narrow band derived from the minimal cut found at the previous coarser level. This pyramidal approach with a Gaussian decimation has proven to be robust, even with a high downscaling factor.

\subsection{Combining Spectral Graph Cuts and Atlas Based Segmentation}

To combine the two methods we first segmented an image using the atlas-based segmentation method. From this segmentation we created the third image used in the spectral gradient as well as the source and sink points needed to initialize the graph cut algorithm. Four hundred sink points are randomly chosen from the brain excluding the area where the left and right thalamus are segmented in the atlas based method. The source points are randomly chosen from $7 \%$ of the atlas-based segmentation. The amount of seed points that were chosen was based upon the observations made in [1]. In [1], different percentages of seed points, taken from the ground truth, were tested and this percentage was shown to result in a good segmentation. We make the assumption that a higher percentage of seeds may cause the graph cut to not be able to correct errors made in the atlas-based segmentation. The amount of sink and source points chosen should also account for a large enough sampling of both the intensities inside and outside of the thalamus for the negative log likelihood (8) (9) to be effective. 


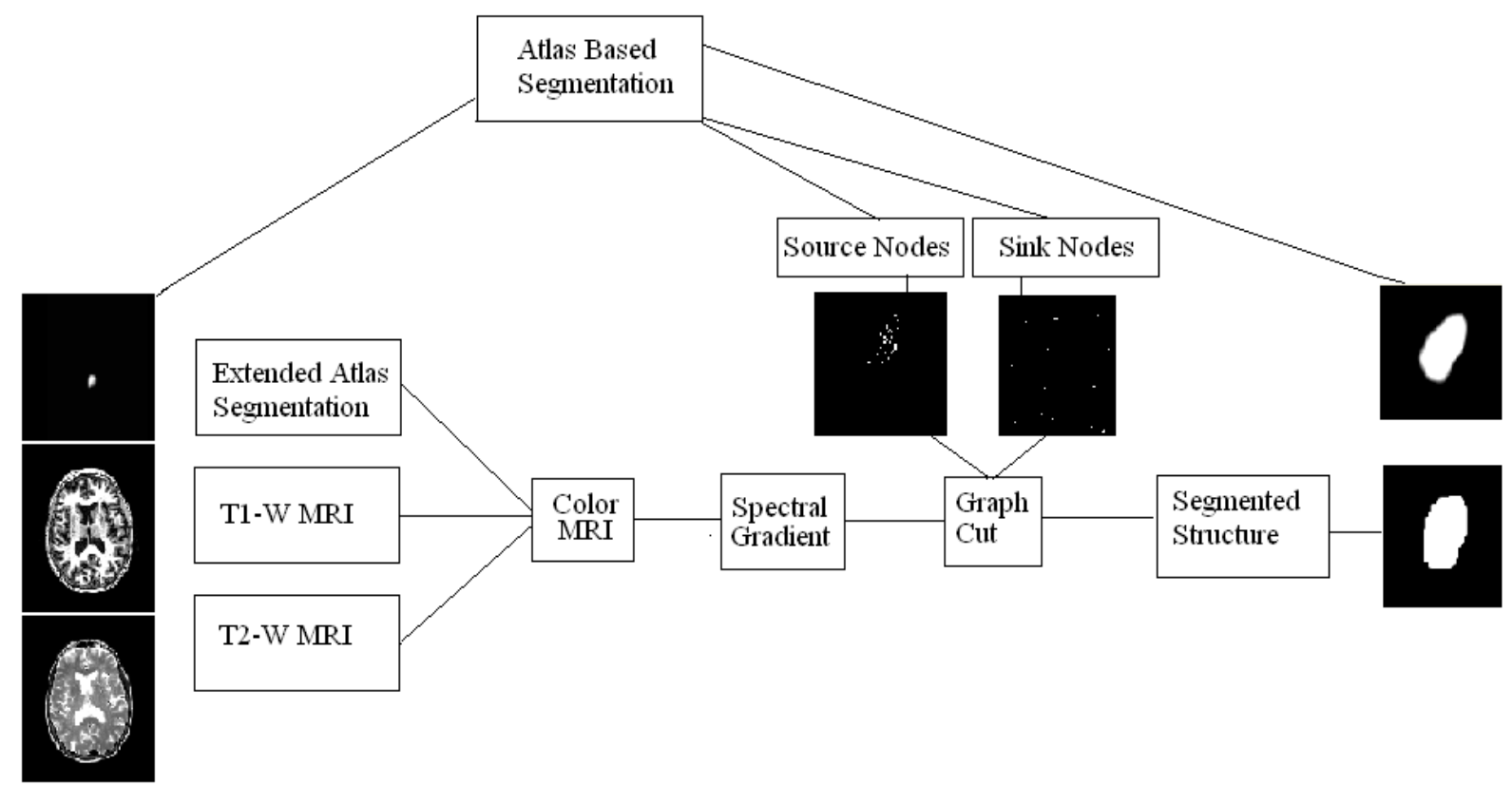

Figure 2: The spectral gradient graph cut segmentation process

The third image is created by taking the actual segmentation results of the atlas-based segmentation method and adding a gradual slope starting $3 \mathrm{~mm}$ inside the edge of the segmentation and continuing to $3 \mathrm{~mm}$ away from the edge to end at zero. Most of the error that may occur when utilizing an atlas-based segmentation should be within this $6 \mathrm{~mm}$ slope. The slope will allow the graph cut algorithm to change the boundary of the segmentation if there is a larger gradient somewhere within the extended atlas-based segmentation, compensating for errors that may occur in the initial segmentation. The segmentation also gives the graph cut algorithm needed spatial knowledge. Without the atlas segmentation input into the process, the graph cut is likely to segment many different tissue classes that resemble the thalamus throughout the brain, yet with the atlas segmentation included it is restricted. If the atlas-based segmentation over or under estimates the size of the target, the gradient of the other two images in the multi-spectral space is, in most cases, sufficient to drive the graph cut segmentation to the actual border of the object. The T1-w, T2-w and atlas-based segmentation are all bounded into an area 60x60x60 voxels wide. This area was chosen on the atlas image and was projected onto the target image. The graph cut algorithm is computed on this reduced image. See Figure 2 for a diagram of the procedure.

\section{RESULTS}

Segmentations were performed using two methods: a pure atlas based segmentation and an automatic combination of the two methods. The results obtained using the Dice Similarity Coefficient (DSC) can be seen in the first column of Figure 3. Segmentations were also performed utilizing three separate atlases to show the sensitivity of the two methods. The first atlas is the original manually segmented atlas while the other two atlases are the original atlas dilated and eroded. The DSC measures can be seen in Figure 3. On average a single segmentation took 417 seconds, 414 seconds for the atlas based method and 3 seconds for the graph cut method. The algorithms were run on a computer with a Quad Core at $2.39 \mathrm{GHz}$ and $3.25 \mathrm{~GB}$ of RAM. 


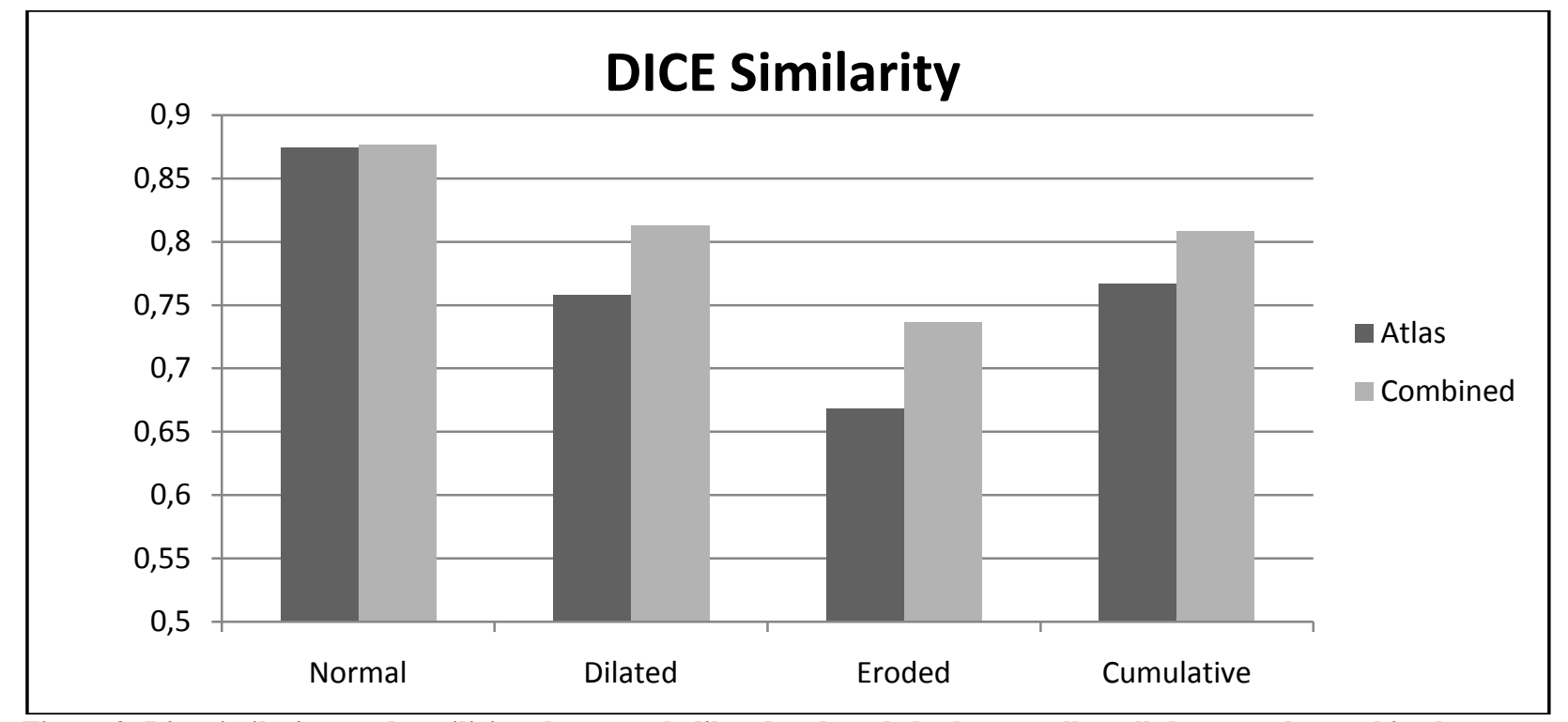

Figure 3: Dice similarity results utilizing the normal, dilated and eroded atlas as well as all three results combined

The atlas-based method segments the thalamus well with an average Dice Similarity Coefficient (DSC) of 0.87. The spectral graph cut method shows similar results with an average DSC measure of 0.88. The atlas based method's DSC value, however, was reduced to 0.76 and 0.67 when dilated and eroded respectively, while the graph cut method retained a DSC value of 0.81 and 0.74 .

A paired t-test was run with the null hypothesis that there is no difference between the results of the atlas-based segmentation method with the normal atlas and the results of the graph-cut algorithm. There is no statistical difference with a $\mathrm{t}=0.308977$ when using the correctly segmented atlas. However, when including the dilated and eroded atlas segmentation results alongside the results of the graph-cut algorithm we find that there is a statistical difference between the results with $\mathrm{t}=2.68 \mathrm{E}-13$.

\section{NEW OR BREAKTHROUGH WORK PRESENTED}

The new work that was presented in this paper is the combination of two segmentation methods: atlas based segmentation and spectral gradient graph cuts. We have automated the graph cut algorithm by seeding the semiautomatic method utilizing the results of an atlas based segmentation. We have also restricted the graph cut to only segment within the extended boundary of the atlas based segmentation. Finally, the combination of the two methods creates a more robust algorithm that can compensate for mistakes made in the manual atlas segmentation.

Excellent work has been previously completed utilizing atlas-based segmentation to include a priori shape information for the segmentation of the hippocampus by Lijn et al [20]. Our method differs, however, since we utilize both T1 and $\mathrm{T} 2$ images. We also keep the classic graph-cut algorithm energy function the same, utilizing the atlas based segmentation as a third image instead of including it in the energy function. Our atlas based segmentation process differs since we define a bounding box for the non-rigid registration resulting in a faster and more accurate atlas segmentation [8], while the work of Lijn et al. [20] uses a global non-rigid registration. Finally, we add a gradually descending slope to the included third image that allows the gradient of the T1 and T2 images to better affect the segmentation.

Freedman et al. [21] included shape information alongside the graph cut method by utilizing a predefined level-set shape template. The method, however, still requires user input to define the sink and source seeds.

Our results are also comparable to other results in the literature [4][5][7], with DSC values of 0.89, 0.87, and 0.89 respectively. Even when utilizing a deformed atlas our results were nearly equal to other methods [6][22]. The framework we utilized is generic and was not optimized specifically for thalamus segmentation. Thus, it can be applied 
to any structure of interest. Since atlas-based segmentation is generally accepted to be a powerful segmentation method [23][24], our approach could be utilized to enhance the robustness of the current atlas-based methods.

\section{CONCLUSIONS}

We have successfully combined the spectral gradient graph cut algorithm and atlas based segmentation method. The atlas based segmentation method automates and gives spatial information to the graph cut algorithm, which refines the atlas based segmentation results. The spectral gradient graph cut method increases the robustness of the atlas based segmentation method that is dependent on the initial manual segmentation of the atlas.

Also, it is likely that a less refined atlas segmentation is possible with the addition of the graph cut algorithm; therefore it may be possible to do a faster registration, increasing the speed of the algorithm. Since the graph cut algorithm's average run time is under 3 seconds, the usage of the algorithm could improve the speed of segmentation.

\section{ACKNOWLEDGMENTS}

This work was partially supported by NSF grant DMS-0334769.

\section{REFERENCES.}

1. Lecoeur, J., Ferré, J.-C., Collins, D. L., Morrissey, S., and Barillot, C., "Multi Channel MRI Segmentation With Graph Cuts Using Spectral Gradient And Multidimensional Gaussian Mixture Model," Proceedings of SPIE Medical Imaging 2009: Image Processing, Pluim, J.-P. and Dawant, B.-M., eds., 7259 (2009).

2. Herline A. J., J. L. Herring, J. D. Stefansic, W. C. Chapman, R, L. Galloway, and B. M. Dawant, "Surface registration for use in interactive image-guided liver surgery" Lecture Notes in Computer Science, Proc. $2^{\text {nd }}$ Med. Image Comput. Computer-Assisted Intervention (MICCAI '99), vol. 1679, pp. 892-899, Cambridge, U.K. (1999)

3. Heckenberg, G. and Xi, Y. and Duan, Y. and Hua, J. and Muzik, O. "Thalamus Segmentation from MRI Images by Lagrangian Surface Flow". International Conference on Engineering in Medicine and Biology (2005).

4. Pohl, K., Fisher, J., Grimson, W., Kikinis, R., and Wells, W., "A bayesian model for joint segmentation and registration," NeuroImage 31, pp. 228-239 (2006).

5. Akhoundi-Asl, A. and Soltanian-Zadeh, H., "Nonparametric entropy-based coupled multi-shape medical image segmentation," International Symposium on Biomedical Imaging, (2007).

6. Akselrod-Ballin, A., Galun, M., Gomori, M.J., Basri, R., Brandt, A. "Prior Knowledge Driven Multiscale Segmentation of Brain MRI," Ayache, N., Ourselin, S., Maeder, A. (eds.) Med. Image Comput. Computer-Assisted Intervention 2007, Part II. LNCS, vol. 4792, pp. 118-126. Springer, Heidelberg (2007).

7. Moghaddam, MJ., Soltanian-Zadeh, H., "Automatic segmentation of brain structures using geometric moment invariants and artificial neural networks," Information Processing in Medical Imaging (2009).

8. Rohde, G., Aldroubi, A., and Dawant, B., "The adaptive bases algorithm for intensity-based nonrigid image registration," IEEE Transactions on Medical Imaging 22(11), pp. 1470-1479 (2003).

9. Studholme, C., Hill, D. And Hawkes, D., "An overlap invariant entropy measure of 3D medical image alignment", Pattern Recognition vol. 32, pp. 71-86, (1999)

10. Wu Z., "Multivariate compactly supported positive definite radial functions," Adv. Comput. Math. 4, pp. 283-292 (1995).

11. Rueckert D., Sonoda L., Hayes C., Hill D., Leach M., and Hawkes D., "Non-rigid registration using free-form deformations: Application to breast MR images," IEEE Trans. Med. Imaging 18, pp. 712-721 (1999).

12. Burr D. J., "A dynamic model for image registration,” Comput. Graph. Image Process. 15, pp. 102-112 (1981).

13. Boykov, Y. and Jolly, M.-P., "Interactive graph cuts for optimal boundary \& region segmentation of objects in ND images," International Conference on Computer Vision, pp. 105-112 (2001).

14. Boykov, Y. and Funka-Lea, G., "Graph cuts and efficient N-D images segmentation," International Journal of Computer Vision 70, pp. 109-131 (2006).

15. Geusebroek, J.-M. and Dev, A. and van den Boomgaard, R. and Smeulders,A. and Cornelissen, F. and Geerts, H. "Color Invariant Edge Detection". Scale-Space Theories in Computer Vision vol. 1252, pp. 459-464 (1999).

16. Koenderink, J., Color Space, Utrecht University (1998). 
17. Lombaert, H., Sun, Y., Grady, L., and Xu, C., "A multilevel banded graph cuts method for fast image segmentation," IEEE European Conference on Computer Vision, Lecture Notes in Computer Science vol. 1842, pp. 331-241 (2000).

18. Karypis, G. and Kumar, V., "Multilevel k-way partitioning scheme for irregular graphs," Journal of Parallel and Distributed Computing vol. 48, pp. 96-129 (1998).

19. Adalsteinsson, D. and Sethian, J.-A., "A fast level set method for propagating interfaces," Journal of Computational Physics vol. 188, pp. 269-277 (1995).

20. van der Lijn, F., den heijer, T., Breteler, M., and Niessen, W., "Hippocamupus segmentation in mr images using atlas registration, voxel classification and graph cuts," NeuroImage 43, 708-720 (2008).

21. Freedman, D. and Zhang, T., "Interactive graph cut based segmentation with shape priors," IEEE Conference on Computer Vision and Pattern Recognition, pp. 755-762 (2005).

22. Scherrer, B., Dojat, M., F., F., and Garbay, C., "LOCUS: LOcal cooperative Unified Segmentation of MRI Brain Scans," International Conference on Med. Image Comput. Computer-Assisted Intervention, pp. 219-227 (2007).

23. Suri, J. and Singh, S. and Reden, L. "Computer Vision and Pattern Recognition Techniques for 2-D and 3-D MR cerebral Cortical Segmentation (Part I): A State-of-the-Art Review". Pattern Analysis and Applications vol. 5, pp. 46-76 (2002)

24. Pham, D. and $\mathrm{Xu}, \mathrm{C}$. and Prince, J. "A Survey of Current Methods in Medical Image Segmentation". Annual Review of Biomedical Engineering vol. 2, pp. 315-337 (2000).

25. Brunetti, A., Postiglione, A., Tedeschi, E., Ciarmiello, A., Quarantelli, M., Covelli, E., Milan, G., Larobina, M., Soricelli, A., Sodano, A., and Alfano, B., "Measurement of global brain atrophy in alzheimer's disease with unsupervised segmentation of spin-echo mri studies.," Journal of Magnetic Resonance Imaging 11(3), pp. 260-266 (2000).

26. Coupé, P., Yger, P., Prima, S., Hellier, P., Kervrann, C., and Barillot, C., "An Optimized Blockwise Non Local Means Denoising Filter for 3D Magnetic Resonance Images," IEEE Transactions on Medical Imaging 27(4), pp. 425-441 (2008).

27. Barra, V. and Boire, J.-P., "Automatic segmentation of subcortical brain structures in mr images using information fusion," IEEE Transactions on Medical Imaging 20(7), pp. 549-558 (2001).

28. Akselrod-Ballin, A., Galun, M., Gomori, M.J., Basri, R., Brandt, A. "Atlas Guided Identification of Brain Structures by Combining 3D Segmentation and SVM Classification," Larsen, R., Nielsen, M., Sporring, J. (eds.) International Conference on Med. Image Comput. Computer-Assisted Intervention. Lecture Notes in Computer Science, vol. 4191, pp. 209-216 (2006).

29. Amini, L. and Soltanian-Zadeh, H. and Lucas, C. and Gity, M. "Automatic segmentation of thalamus from brain MRI integrating fuzzy clustering and dynamic contours number". IEEE Transactions on Biomedical Engineering vol. 51, pp. 800-811 (2004). 